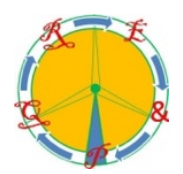

\title{
Study of the requirements of an autonomous system for surface water quality monitoring
}

\author{
M. Barros, P. Granchinho, C. Ferreira, P. Neves, H. Magalhães, L. Santos, B. Lopes, J. Marques \\ H. Pinho ${ }^{1}$, S. Mourato ${ }^{2}$ and A. Martins ${ }^{3}$ \\ ${ }^{1}$ Departmental Engineering Unit - Smart Cities Research Center (Ci2) \\ E.S.T.T., Institute Polytechnic of Tomar (IPT) \\ Quinta do Contador - Estrada da Serra, 2300-313 Tomar (Portugal) \\ Phone: +351 249328 100, Fax number: +351 249328 186, e-mail: fmbarros@ipt.pt, granchinho@ipt.pt,
}

cferreira@ipt.pt, pedroneves@ipt.pt, hpinho@ipt.pt, Isantos@ipt.pt, $\underline{\text { bablopes@ipt.pt, jomarques@ipt.pt }}$

${ }^{2}$ Institute Polytechnic of Leiria (IPL)

Phone: +351 244830 010, e-mail: ipleiria@ipleiria.pt

${ }^{3}$ Intermunicipal Community of Médio Tejo (CIMT)

Phone: +351 249730 060, Fax number: +351 249730 069, e-mail: geral@cimt.pt

\begin{abstract}
In recent years, there has been increasing awareness of the preservation, protection and sustainable use of natural resources. Water resources, being one of the most important natural resources, face major threats due to contamination by pollutants of various types and origins. Maintaining the quality of water resources requires more robust, reliable and more frequent monitoring than traditional techniques of data collection based on sporadic, discontinuous and manual processes. The management of large geographical areas, the insufficient spatiotemporal discretization of the values of samples collected by traditional processes and the unpredictability of natural phenomena, require a new approach to data collection procedures. This article, which is the result of ongoing research, defines the technical requirements and technologies used in a continuous and regular monitoring of surface water quality in freshwater systems, whose data acquisition system helps to identify the sources of pollution and the contaminants flow along the waterways. The design of a versatile real-time water quality monitoring system, which, due to its environmental constraints should be based on renewable energies and wireless transfer of energy, will contribute to improve the management and effective protection of water resources.
\end{abstract}

\section{Keywords}

Water quality monitoring, wireless sensor networks, wireless charging, autonomous vehicles, environment protection, resources management.

\section{Introduction}

The Directive 2000/60/EC of the European Parliament and of the Council, of 23 October 2000, establishes a framework for community action to the preservation, protection and sustainable use of water resources and a set of regulations that define, inter alia, the data collection in processes for monitoring the status of surface waters. However, these procedures are out of step with the threats, challenges and unpredictability that water ecosystems face today.

The discontinuity or irregularity of the data acquisition, as well as the temporal discretization of the values, make them not enough to monitor the effects of certain phenomena on the quality of the water bodies, establishing an obstacle to finding out their causes or to describe the evolution of those phenomena over time.

In case of discharge of polluting effluents into the aquatic environment, water quality parameters may change significantly in a short time. Information gaps in water quality monitoring data produce delays that make it difficult the detection of events and sources of pollution. For this reason, there should be frequent monitoring. The lack of reliable and comparable data is an obstacle to the development of an effective monitoring and surveillance system in case of environmental disaster.

Conventional processes involve the manual collection of samples from various points in the sampling network, followed by laboratory testing and analysis. These methods can be considered ineffective as they are cumbersome, time-consuming, and lack real-time results to promote a proactive response to water contamination [1]. The acquisition of data in aquatic ecosystems becomes even more difficult or unfeasible when covering large areas or with difficult access.

The present work aims to develop a system that solves the need for monitoring the surface water quality in natural and artificial freshwater systems of different types, in a continuous and regular way. It is intended to proceed with the acquisition of quality data in order to identify sources of pollution or to estimate the discharge and dispersion of 
contaminants along watercourses. The design of a versatile real-time water quality monitoring system contributes to the management and protection of water resources thus it can increase security in the water supply of populations and turn the resources management more efficient and sustainable.

A physical prototype, typically an aerial or water electric vehicle, has energy needs that must be considered. To make an environmentally friendly system, its energy typically must come from generation by the water stream (where it exists) through a hydro generator or, generally, from sunlight.

The prototype will operate in an environment with high levels of humidity and potentially "dirty", so the possibility of battery recharging without electrical contact, commonly called "wireless charging", has to be considered.

\section{Continuous and real-time water quality monitoring systems}

This section provides an overview of the possible techniques to be used in environmental monitoring, especially those concerning surface water status. The most relevant solutions include environmental sensor networks and those based on environmental robotics.

\subsection{Sensor Networks}

Environmental Sensor Networks (ESN) have recently emerged as a promising technology for the environmental monitoring in aquatic environments [2]. ESN's consist of a group of small networked devices that allow real-time monitoring of relevant variables at various fixed and predetermined points [3]. ESNs have the advantage of allowing data collection in multiple locations simultaneously [4]. Despite their potential, they also face several technical challenges and limitations. A major limitation of ESNs is that they usually need to have fixed sampling locations [5] and therefore do not have the ability to self-reconfigure in response to unexpected events or to increase coverage in the area of interest.

\subsection{Environmental Robotics}

Robotic systems are increasingly being used as fundamental data-gathering tools [5]. Robotic systems for environmental monitoring are a promising solution to overcome the limitations of ESNs using robotic platforms that can add mobility to sensor networks and increase the full potential of ESNs. One obvious advantage of utilizing robotics in environmental sciences is that they allow the monitoring and sampling of events that are too dangerous, or impossible, for humans to undertake. Environmental robotics has been subject to significant progress in recent years. Cooperative robotic teams can collect data from several places simultaneously, allowing dynamically the increase the spatial and temporal areas of data collection that would be impossible to achieve with a single robot or a network of static sensors [5]. Let's briefly review, some of the robotic systems used in environmental monitoring.

\subsubsection{Autonomous surface vehicle}

Autonomous Surface Vehicles (ASV) are robotic vehicles that move on the surface of the waterbody and record a series of data related to environmental monitoring and others such as underwater mapping, etc. It is an alternative method of data collection that goes beyond the limitations of fixed and floating satellite systems and networks used by scientists and researchers. Some examples of this technology include the ROAZ II system [6] developed at the Polytechnic Institute of Porto (ISEP), designed to operate in aquatic environments such as the study and exploration in oceans, rivers and reservoirs. The THA1592 project implemented in the IPT had an autonomous surface vehicle [7], with the configuration of an inverted catamaran and it was composed of an ASV and a UAV [8].

\subsubsection{Unmanned Air Vehicle}

Unmanned Air Vehicles (UAVs) are often unable to achieve continuous operation because of their limited movement and energy dependence. One of the solutions can be achieved through the collaboration of several UAVs to maintain the objective of continuous operation. In [9] and [10] two solutions are presented based on aerial vehicles used in applications of sampling of water in remote sites.

\subsubsection{Autonomous Underwater Vehicle}

An Autonomous Underwater Vehicle (AUV) equipped with a specific environmental sensor may be a good choice for deep water monitoring. Today, AUVs are not only technologically advanced but also commercially available, and they can be able to recognize large areas in a short period of time. Underwater pollution requires on-site sampling and measurement techniques so, the existing remote monitoring techniques are efficient and developed for in-depth environmental disasters.

\subsection{Technology Comparison}

There are a range of solutions, techniques and strategies that have been scientifically and commercially implemented in monitoring and environmental control applications. In general, autonomous robots have the potential to overcome the limitations inherent in ESNs. However, new methods are needed to allocate resources to achieve various observation objectives and to allow efficient control of large groups of cooperative mobile detection systems, [11] and [12].

\section{SAM-QAS system requirements}

This section summarizes the work done so far in defining the requirements of an autonomous system for surface water quality (SAM-QAS) monitoring aimed at identifying the sources of pollution and the contaminants flowing along the waterways. In order to achieve the proposed objectives it is essential to develop a requirements specification plan, which will serve as a guide for implementing a new approach that, taking advantage of technological advances and new communication systems, will overcome the traditional technical limitations of monitoring and data collection in water environments.

In a first step, a review of the literature on water quality monitoring and wireless sensor network (WSN) was carried out, which allowed to determine the fundamental aspects and to formulate the new approach specifications. 
These consist on defining or specifying the following parameters: (i) the environmental variables and the quality parameters to be measured; (ii) the recording frequency, ie when and how often the variables should be acquired; iii) the monitoring area for the knowledge of the local conditions and system preconditions; iv) the action range of the autonomous system and its autonomy; v) the use of open standards-based communication protocols to ensure transparent communication of information between system modules; and vi) the user interface that specifies the format, presentation and storage of data.

\section{A. Water quality parameters to analysed}

The SAM-QAS system was specified to allow the acquisition of a specific set of environmental data that characterize the aquatic environment and help to detect variations (deviation) in water quality. In the study of chemical and/or physical parameters, it was considered that water temperature, although only a generic indicator of water quality, represents relevant information to identify deviations and to allow correlation with other parameters. The second parameter to analyse is the $\mathrm{pH}$ of the water, which allows detecting an industrial pollution point (mineral acidity) or water eutrophication processes (relation between the oxidation of organic matter and the $\mathrm{CO}_{2}$ content). The analysis of other parameters values may be planned to complement surface water characterization, such as the measurement of dissolved oxygen, redox, conductivity, salinity and dissolved solids.

\section{B. Data acquisition zone}

The Castelo do Bode reservoir in Portugal has been adopted in this work, as data acquisition zone and for the development and testing of the system, due to its geographical proximity. It is the main national water reservoir for public supply and it has a maximum extension of $60 \mathrm{~km}$. The Castelo do Bode reservoir occupies an area of about 3300 ha. It has a total storage capacity of about $1100 \mathrm{hm}^{3}$ and a useful capacity of $900 \mathrm{hm}^{3}$. It has been a study subject through a brief characterization to know the previous conditions and to identify the external factors that can influence the system development. The mapping of the Castelo do Bode reservoir was done with the survey of the main tributaries, in order to understand the spatial distribution of the areas of data acquisition interest. The size of the system and the requirements it must have in order to obtain temporal and spatial large-scale values, can be perceived by the distribution and extension of the study area in Figure 1. The greatest interest areas of this work include: (1) water catchment points for human consumption because they are the areas where water quality is monitored and where there may be an interest in comparing samples that have been acquired over time, at these stations; 2) the tributaries that may contribute with more domestic or industrial polluting load to the reservoir. The downstream zones of the respective confluences with the Zêzere river are relevant for the acquisition of data because the effects of effluent loading can be monitored over time. In case of a rapid variation in the analysed parameters, a rapid and timely detection of a source of pollution can be possible, and 3) population clusters on the banks are also considered risk areas of point pollution, because although the number of inhabitants is very small, the reservoir has lentic characteristics, which reduces its capacity for self-purification.

In the summer the population significantly increases in those clusters, rising the pollutant load produced. The lower flow tributaries, which have significant seasonal variations, tend to increase the concentration of the pollutant load in the summer months due to the reduction of their flow. [13].

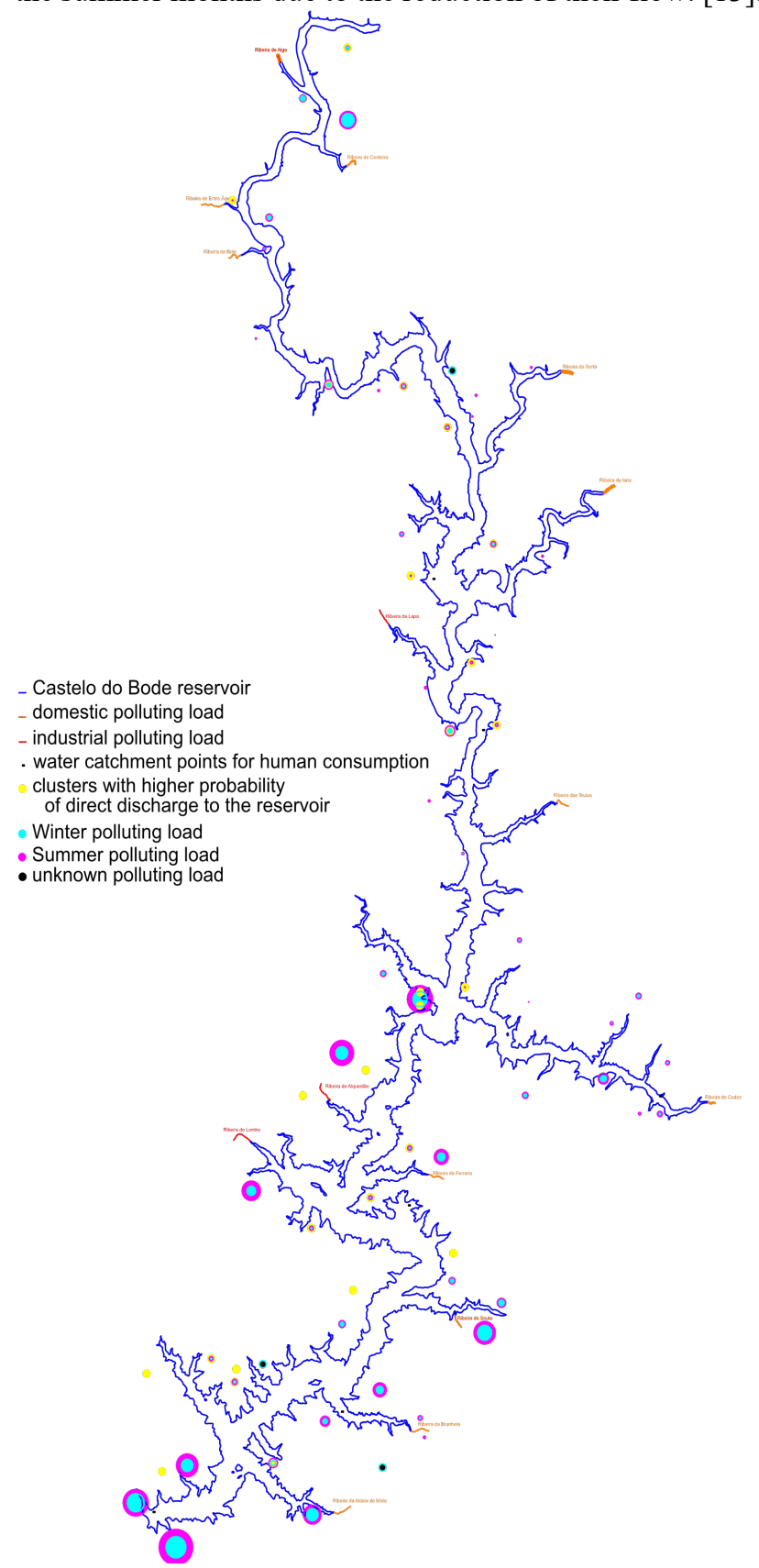

Figure 1 - Distribution of the areas at greatest risk of pollution of the Castelo do Bode reservoir

\section{Action range of the autonomous system}

The system must be versatile and allow autonomous adaptation of its missions according to the scenarios of a possible source of pollution. Therefore, the action range of a mission must be scalable depending on the autonomy of the device, and it can be programmed remotely or from a collaborative intelligent strategy processed locally among 
the robots. The preferential research area should consider the most critical areas presented in section B of this chapter.

\section{Registration frequency}

The acquisition of real-time data is an essential condition in this type of applications, as it will allow the immediate detection of pollution points and a timely action on it. The data collected by the system have different needs in relation to the sampling period, so the system must be able to configure the recording frequency of each sensor according to its purpose. For example, the temperature can be set to collect data samples every 5 minutes or when explicitly requested.

\section{E. Autonomy}

In order to ensure the continuity and regularity of available data and to reduce system maintenance times and costs, it will be necessary to ensure the autonomous operation of the power supply system. It can be made by using redundant data and energy storage systems, standalone charging systems and independent power conversion and storage systems. This will typically consist of an array of photovoltaic panels with an interface through an electronic power converter that implements a Maximum Power Point Tracking (MPPT) algorithm and allows the charging of a set of batteries.

The batteries of autonomous vehicles would thus be recharged by accessing a specific point for energy transfer. Due to the surrounding environment, which is typically damp and dirty, a charging station with inductive-resonant power transfer would be most appropriate [14].

\section{F. Communication Protocols}

In order to guarantee the continuity and regularity of the data available and the interoperability between the various modules of the system, open communication protocols based on Internet of Things (IoT) standards should be used.

\section{G. User interface}

The data visualization tool along with analytical tools plays an important role in this system. Thus, a graphical user interface is being developed, taking advantage of the latest IoT data visualization platforms that allow a pleasant and intuitive interaction with the data. Besides, it enables the data anomalies diagnose and report and it activates alarms in case of a possible pollution point. The system will be able to provide data regarding the system's operation.

\section{Development of Robotic water quality monitoring system}

This section presents an overview of the SAM-QAS architecture and describes its main components. To accelerate the development phase, a simple but robust robotics platform was selected. The communications infrastructure was built in order to take advantage of opportunities and benefits that cloud services offer.

\subsection{Architecture}

Figure 2 presents, schematically, the three main functional blocks of the proposed system: a mobile robotics platform, an IoT gateway platform and the cloud-based data storage and retrieval. For simplicity, the two systems will be functioning separately in a complementary way. The robotic modules will allow the vehicle to execute userdefined missions, while the gateway base station gathers and processes the data from the attached sensors and bridges the communication between the robotic sensing devices and the cloud-based systems. Web-analytics dashboards help to generate useful insights making it possible to identify contamination (pollution) sources and other alerts, functioning as a decision-making tool to trigger the necessary environmental protection mechanisms.

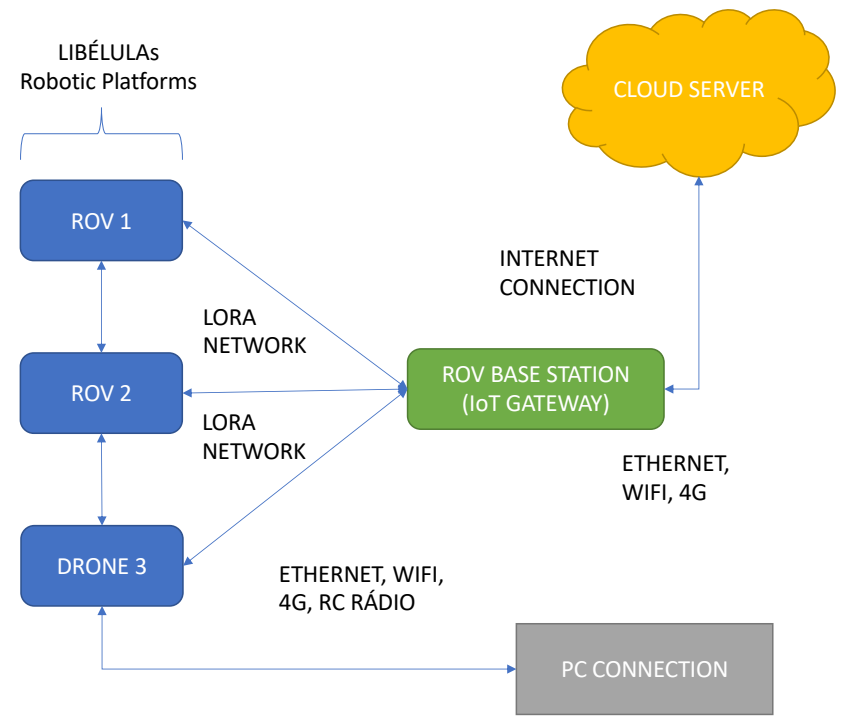

Figure 2 - Integration of robotics, IoT and cloud services technologies

\subsection{Robotic platform overview}

The system consists of an autonomous, mobile and robotic monitoring system unit (called LIBÉLULA, in English means DRAGONFLY) with onboard sensors for the acquisition of water quality parameters $(\mathrm{pH}$, temperature, conductivity, etc.), a long-range wireless radio communication module and a microcontroller board with a GPS and compass unit (Fig. 3).

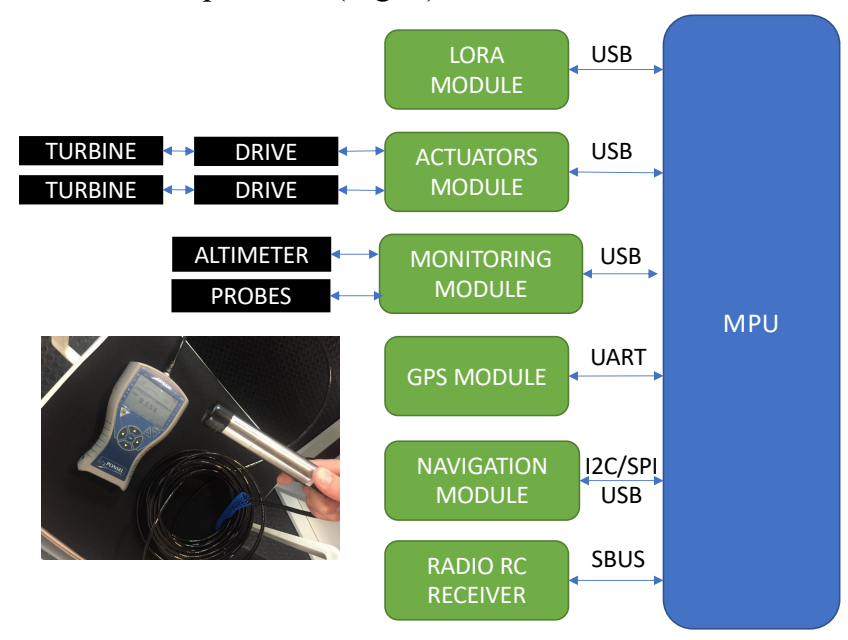

Figure 3 - The LIBELULA search unit

Each LIBÉLULA allows remote monitoring of relevant water quality data and their dissemination in real time to a 
central server and cloud platforms [7]. The core of each robotic system is a Micro Processor Unit (Raspberry Pi 3 $\mathrm{b}+$ ) running Linux Ubuntu Core operating system. All the system is developed using ROS (Robot Operating System) which is a set of Open-Source software libraries, drivers and tools powered by a communication system of Publish/Subscribe messages. Each functionality (Monitoring, Navigation, Communication, etc.) was developed as an electronic module with a $32 \mathrm{bit} \mathrm{MCU}$, FreeRtos and UART/USB interface for the low-level/Real Time programming. These modules are integrated on ROS as a Software Node. The distributed architecture allows an easier integration with the MPU's (USB interface without low-level programming) and the ability to rearrange the setup if needed.

\subsection{IoT Gateway Platform overview}

The IoT Gateway Platform illustrated in Fig. 4, is a Remote Operated Vehicle (ROV) Base Unit built with the objective of working as an IOT Gateway (Border/Edge Router). The IoT ROV Base Unit acquires the data monitored by the Mobile Search Units through LORA wireless Technology (Long Range Low Power wireless communication Protocol LPWAN), storages the information and forwards the data to a Cloud Web Server through an Ethernet Connection or Cellular Communication like $3 \mathrm{G} / 4 \mathrm{G}$. The core system also developed with ROS gives this unit the ability to be a "Mobile Gateway" allowing flexibility on the monitoring areas and integrating new technology/modules.

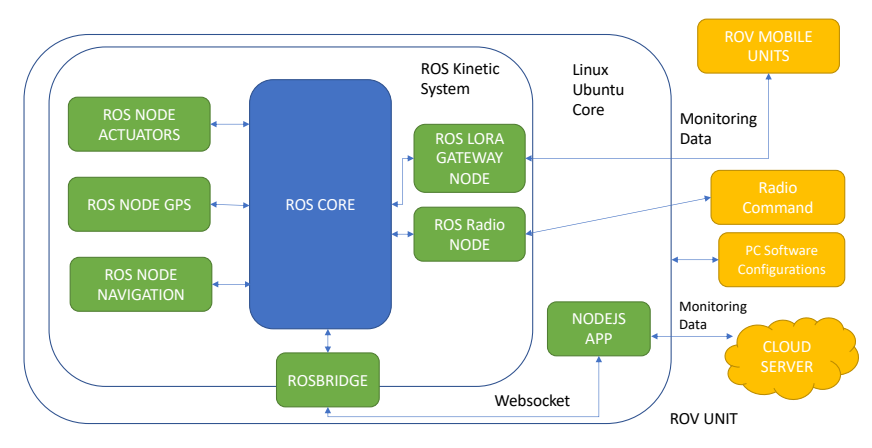

Figure 4 - Block diagram of the IoT ROV Base unit

\subsection{IoT Cloud Platform overview}

Cloud computing, on-demand computing or Internet based computing is a low-cost technology that offers fast and reliable processing, data storage, and several developer and analytical tools that allow both easy integration of physical devices and advanced data analysis.

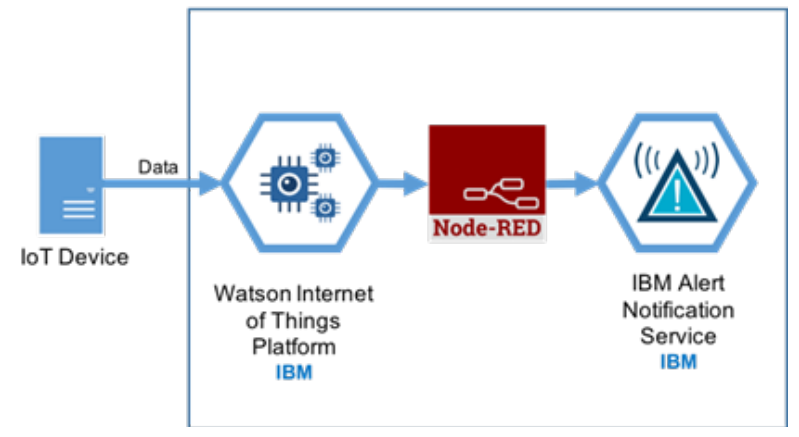

Figure 5 - Data flow for IoT system in the cloud (source: IBM)
In this work, cloud computing services are to be used as the infrastructure backbone for the system as they provide fast deployment, continuous service, security and scalability. Although any provider of cloud services can be used, Figure 5 shows a diagram consisting of an interconnected suite of applications made available from IBM, specifically for IoT.

\section{Conclusions}

Today, reservoirs, rivers and lakes face major threats due to contamination by pollutants of various types and origins, causing frequent fluctuations in ecosystem equilibrium conditions. Therefore, the need to implement a large-scale monitoring system that is effective and sensitive to such changes is justifiable.

The acquisition of real-time data is an essential condition in this type of applications, as it will allow the immediate detection of pollution points and a timely action on it. The available data continuity and regularity will allow the support of the aquatic environment studies and the forecasting systems modelling of future conditions, guiding the decision processes.

The present work defines the technical requirements of a capable system of surface water quality monitoring in natural and artificial freshwater systems of different types, in a continuous and regular way. The acquired data allows to identify sources of pollution or to estimate the discharge and dispersion of contaminants along watercourses.

The project is a versatile, robust and reliable real-time water quality monitoring system, thus contributing to the management and protection of water resources, increasing security in the water supply of populations and a more efficient and sustainable management of water resources.

\section{Acknowledgement}

This work was supported by Centro2020, Portugal 2020 and European Union (EU) under the grants, CENTRO-010145-FEDER-024052E - Libélula: Mobile robotic surface water quality monitoring system.

\section{References}

[1] M. Pule, A. Yahya and J. Chuma, "Wireless sensor networks: A survey on monitoring water quality", in Journal of Applied Research and Technology, 2017, 15, pp. 562-570.

[2] A. Ballesteros-Gomez and S. Rubio, "Recent Advances in Environmental Analysis," in Analytical Chemistry, 2011, Vol. 83, 12, pp. 4579-4613.

[3] M. Dunbabin, and L. Marques, "Robots for environmental monitoring: Significant advancements and applications" in IEEE Robotics and Automation Magazine, 2012, Vol. 19, pp. 24-39.

[4] G. Xu, W. Shen, and X. Wang, "Applications of Wireless Sensor Networks in Marine Environment Monitoring: A Survey," Sensors, 2014, Vol. 14, pp. 16932-16954.

[5] M. Dunbabin and L. Marques, "Robots for Environmental Monitoring: Significant Advancements and Applications," in 
IEEE Robotics and Automation Magazine, 2012, Vol. 19, 1, pp. 24-39.

[6] H. Ferreira, C. Almeida, A. Martins, J. Almeida, N.Dias and A. Dias, "Autonomous bathymetry for risk assessment with ROAZ robotic surface vehicle" Proceedings of the OCEANS '09 IEEE conference, Bremen, Germany, 2009.

[7] D. Raimundo, T. Leite, A. Silva, Projeto de um Veículo Autónomo de Superfície para Monitorização de Águas Fluviais Projeto EEC, IPT, Tomar, 2016.

[8] F. Marques, R. Mendonça, E. Pinto, P. Rodrigues, P. Santana, "A Critical Survey on Marsupial Robotic Teams for Environmental Monitoring of Water Bodies" Proceedings of the OCEANS '15 IEEE conference, Genova, Italy, 2015.

[9] "An open-source watertight unmanned aerial vehicle for water quality monitoring" Proceedings of the OCEANS ' 15 MT/IEEE conference, Washington, DC, USA, 2015.
[10] C. Koparan, Unmanned Aerial Vehicle (UAV) - Assisted Water Sampling, All Theses, Clemson University, 2016.

[11] P. Jiang, H. Xia, Z. He, Z. Wang, "Design of a Water Environment Monitoring System Based on Wireless Sensor Networks" Sensors 2009, 9, pp. 6411-34.

[12] Li, T.; Xia, M.; Chen, J.; Zhao, Y.; de Silva, C., "Automated Water Quality Survey and Evaluation Using an IoT Platform with Mobile Sensor Nodes” Sensors 2017, 17, pp. 1735.

[13] Revisão do Plano de Ordenamento da Albufeira de Castelo do Bode, Projecto de plano, Relatório síntese, July 2002, pp. 12 18 .

[14] M. Simica, C. Bila, V. Vojisavljevicb, "Investigation in wireless power transmission for UAV charging" Procedia Computer Science 2015, 60, pp. 1846 - 1855 